\title{
Cooperative Guidance Law with Predefined-Time Convergence for Multimissile Systems
}

\author{
Liang Jing $\left(\mathbb{D},{ }^{1}\right.$ Changzhu Wei $\mathbb{D}^{1},{ }^{1}$ Liang Zhang $\mathbb{D}^{2},{ }^{2}$ and Naigang Cui $\mathbb{D}^{1}$ \\ ${ }^{1}$ Department of Aerospace Engineering, Harbin Institute of Technology, Harbin 150001, China \\ ${ }^{2}$ Department of Aerospace Engineering, Sun Yat-sen University, Guangzhou 510275, China \\ Correspondence should be addressed to Changzhu Wei; weichangzhu@hit.edu.cn
}

Received 10 March 2021; Accepted 25 June 2021; Published 6 July 2021

Academic Editor: Abdellatif Ben Makhlouf

Copyright (C 2021 Liang Jing et al. This is an open access article distributed under the Creative Commons Attribution License, which permits unrestricted use, distribution, and reproduction in any medium, provided the original work is properly cited.

To intercept the maneuvering target through multimissile cooperation, predefined-time cooperative guidance (PTCG) law is presented with constraints including the impact time and the line-of-sight (LOS) angle. In order to achieve simultaneous interception, we propose a PTCG law in the LOS direction based on a predefined-time consensus protocol, which guarantees the achievement of consensus on each missile's impact time within the predefined time. Furthermore, to ensure the predefined-time convergence of the LOS angle and the predefined-time convergence of the LOS angular rate, a PTCG law with a fixed-time disturbance observer (FxTDO) is presented in the normal direction of the LOS. Compared with the traditional finite-time or fixedtime cooperative guidance laws, the proposed PTCG law predefines the upper bound of the settling time as an explicit parameter. Finally, the simulation results of the PTCG law verify the efficiency of the proposed method.

\section{Introduction}

In recent years, with the continuous development of modern military technology, the maneuverability of the target has been remarkably improved, and thus the single-missile combat mode has become more difficult. To overcome this problem, the multimissile cooperative interception is put forward, which has received extensive attention $[1,2]$.

The cooperative interception guidance law requires stringent impact time constraints for multiple missiles to achieve simultaneous arrival. Driven by the requirement, two methods are adopted to complete the cooperative interception mission: the individual homing guidance law [1-14] with the impact time constraint and the cooperative guidance law [15-20]. The individual homing guidance law does not consider communication among missiles. To simultaneously reach the target position, Jeon [4] first proposed the impact time control guidance law (ITCG), which uses the error feedback of the impact time to adjust the coefficient of proportional navigation guidance. Then, a new ITCG was designed in [6], which combines the impact time error feedback with the sliding mode control. Nevertheless, in the aforementioned guidance laws, the impact time is predefined and each missile hits the target according to its own guidance law. Hence, the impact time is not adjustable in flight.

By contrast, the cooperative guidance law deals with the problem of cooperative interception involving communication among multiple missiles. In [15], based on the leaderfollower scheme, a leader-oriented cooperative guidance law was proposed. The leader can coordinate the impact time of followers by the cooperative guidance law. But this method requires high real-time quality and high reliability for the communication of the leader. To tackle this problem, the distributed cooperative guidance law was presented in [16-20], which can relax the topological communication requirement and has stronger robustness. To improve the information transmission capacity, consensus protocols for multiagent systems [21-23] were introduced into the design of distributed cooperative guidance. By utilizing the consensus protocol algorithm, agents can reach a consensus based on local information. To guarantee the multiple missiles achieve a consensus at a faster convergence rate in the cooperative interception missions, the finite-time consensus was put 
forward. In [24], Lv presented a distributed finite-time cooperative guidance (FTCG) law with angle constraints, which utilizes the finite-time consensus to improve the convergence rate and robustness. Then, the FTCG law with an acceleration saturation constraint was proposed in [25], which is characterized by its fast and accurate convergence of the guidance system. Furthermore, since the acceleration of the target is unmeasurable during the flight, it could be considered as a disturbance. And the influence of the unmeasurable disturbance can be reduced by the disturbance observer. In [26], Guo and Liang presented a nonsingular fast terminal sliding mode cooperative guidance algorithm without the target maneuver information, where the unknown target acceleration is estimated by a finite-time disturbance observer. Moreover, a new FTCG law based on the finite-time consensus protocol with LOS angle constraints was presented in [27], which uses an extended state disturbance observer. Then, the FTCG law [27] was introduced into a three-dimensional (3D) multimissile guidance system in [28]. However, for the disturbance observers and guidance laws in [24-29], their convergence time is affected by initial conditions and increases unboundedly as the initial errors increase, which dramatically affects the accuracy of the system.

Fixed-time control was put forward and gained increasing attention following finite-time control. For a fixedtime stable system, a constant independent of initial conditions can uniformly bound the system's convergence time. In the existing research literature, fixed-time control schemes were adopted to design the cooperative guidance law because fixed-time stability enjoys fast convergence and strong robustness. In [30], a distributed fixed-time cooperative guidance (FxTCG) law with an FxTDO was proposed, which guarantees that each missile can reach a consensus within the fixed time. Subsequently, Zhang and Ma [31] developed an adaptive FxTCG law with LOS angle constraints. However, the negative power term exists in the consensus protocol in [31]. To solve this problem, a new FxTCG law was presented in [32], which utilizes an adaptive fixed-time convergence reaching law together with a novel fixed-time consensus protocol. Moreover, Chen et al. [33] proposed a 3D FxTCG law with LOS angle constraints for maneuvering target interception, which can achieve faster convergence and high precision. Lin et al. [34] presented a 3D FxTCG law with impact angle constraints for the simultaneous arrival problem, which can set the desired impact time in advance. However, the selection of fixed convergence time is not arbitrary, and there is usually a complex relationship between the tunable parameters and the fixed convergence time.

To overcome the above drawbacks, Sanchez-Torres originally proposed the predefined-time stability theory in [35]. Predefined-time stability has an obvious advantage in the system settling time. The settling time's upper limit can be arbitrarily set by choosing appropriate controller parameters. Predefined-time sliding mode controllers for dynamic systems were designed in [36-38]. Subsequently, the predefined-time control was used for controlling the attitude of rigid spacecraft and tracking robotic manipulators in $[39,40]$. Besides, a novel predefined-time consensus protocol was proposed in [41], which ensures a predefinedtime consensus for static topologies.

Motivated by the mentioned above, this paper is dedicated to deducing a PTCG law with the LOS angle constraint as well as the impact time constraint. The main contributions are summarized below:

(1) A novel formulation of the predefined-time convergence is proposed. The parameter setting of the given predefined-time convergence is more flexible and convenient, and the limit of the settling time is treated as an explicit tuning parameter.

(2) A new predefined-time consensus protocol is presented with strict proof. And in the LOS direction, according to the above protocol, a PTCG law is proposed for multimissile intercepting the target simultaneously on the basis of predefined-time convergence as well as the given algebraic graph.

(3) In the normal direction of LOS, a PTCG sliding mode control law with an FxTDO is presented to ensure that the LOS angle and the LOS angle rate can realize predefined-time convergence. Considering the uncertain disturbance, an FxTDO is used to estimate the disturbance and compensate for the PTCG law.

The remainder of this paper is structured as follows: Section 2 introduces the cooperative guidance model and preliminary lemmas. A PTCG law is presented with strict proof in Section 3. The numerical simulation results and analysis are given in Section 4. Section 5 concludes this paper.

\section{Problem Formulation and Preliminaries}

2.1. Problem Formulation. Considering $n$ missiles intercept a maneuvering target simultaneously. Figure 1 shows a $2 \mathrm{D}$ planar engagement. The subscript $i,(i=1,2, \ldots, n)$ denotes the $i$-th missile. $M_{i}$ and $T$ indicate the missiles and target, respectively. $X O Y$ is the inertial frame.

The relative kinematic model is given by

$$
\begin{aligned}
\dot{r}_{i} & =V_{T} \cos \left(q_{i}-\theta_{T}\right)-V_{M i} \cos \left(q_{i}-\theta_{M i}\right), \\
r_{i} \dot{q}_{i} & =-V_{T} \sin \left(q_{i}-\theta_{T}\right)+V_{M i} \sin \left(q_{i}-\theta_{M i}\right), \\
\dot{\theta}_{M i} & =\frac{a_{M i}}{V_{M i}} \\
\dot{\theta}_{T} & =\frac{a_{T}}{V_{T}}
\end{aligned}
$$

where $V_{M i}$ and $V_{T}$ indicate the velocity of the missiles and the target, respectively. $r_{i}$ is the distance from the missile to the target. $\theta_{M i}$ and $\theta_{T}$ denote the flight path angles of the missile and the target, respectively. The LOS angle is denoted as $q_{i} . a_{M i}$ and $a_{T}$ represent the $i$-th missile's and the target's normal accelerations.

The derivatives of equations (1) and (2) can be obtained as 


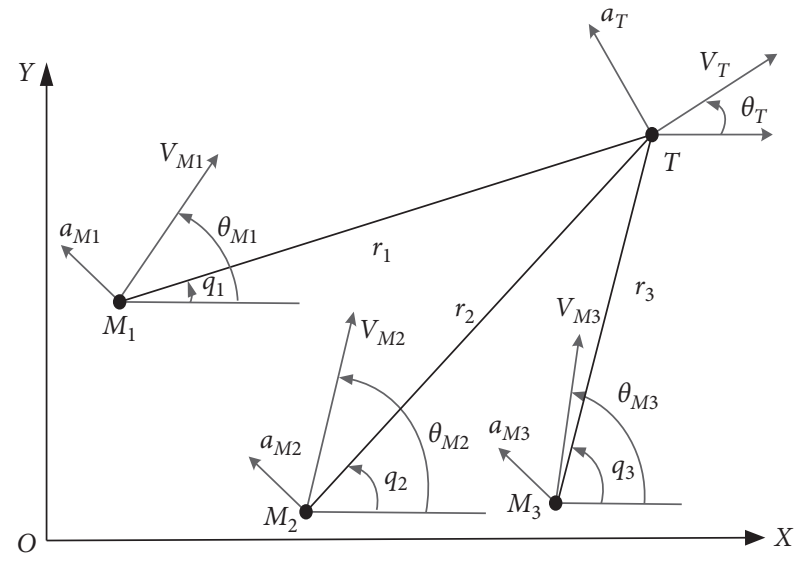

Figure 1: Planar guidance geometry.

$$
\begin{aligned}
& \ddot{r}_{i}=r_{i} \dot{q}_{i}^{2}+u_{r i}-w_{r i}, \\
& q_{i}=-\frac{2 \dot{r}_{i} \dot{q}_{i}}{r_{i}}-\frac{u_{q i}}{r_{i}}+\frac{w_{q i}}{r_{i}},
\end{aligned}
$$

where $u_{r i}$ and $u_{q i}$ are the $i$-th missile's acceleration components along the LOS direction and the normal direction of the LOS. $w_{r i}$ and $w_{q i}$ are the acceleration components of the target along the LOS direction and the normal direction of the LOS. In the actual interception mission, $w_{r i}$ can be considered as zero.

Define $x_{1 i}=r_{i}, x_{2 i}=\dot{r}_{i}, x_{3 i}=q_{i}-q_{f i}$, and $x_{4 i}=\dot{x}_{3 i}=\dot{q}_{i}$. Then, the guidance system is given as

$$
\left\{\begin{array}{l}
\dot{x}_{1 i}=x_{2 i}, \\
\dot{x}_{2 i}=x_{1 i} x_{4 i}^{2}-u_{r i}, \\
\dot{x}_{3 i}=x_{4 i}, \\
\dot{x}_{4 i}=-\frac{2 x_{2 i}}{x_{1 i}} x_{4 i}-\frac{u_{q i}}{x_{1 i}}+\frac{w_{q i}}{x_{1 i}},
\end{array}\right.
$$

where

$$
t_{g i}=-\frac{r_{i}}{\dot{r_{i}}}
$$

Differentiating equation (6) yields

$$
\dot{t}_{g i}=-1+\frac{x_{1 i}^{2} x_{4 i}^{2}}{x_{2 i}^{2}}-\frac{x_{1 i}}{x_{2 i}^{2}} u_{r i} .
$$

Consider $t_{g i}$ as a new state variable, and the dynamic equations are rewritten as

$$
\left\{\begin{array}{l}
\dot{t}_{g i}=-1+\frac{x_{1 i}^{2} x_{4 i}^{2}}{x_{2 i}^{2}}-\frac{x_{1 i}}{x_{2 i}^{2}} u_{r i}, \\
\dot{x}_{3 i}=x_{4 i}, \\
\dot{x}_{4 i}=-\frac{2 x_{2 i}}{x_{1 i}} x_{4 i}-\frac{u_{q i}}{x_{1 i}}+\frac{w_{q i}}{x_{1 i}} .
\end{array}\right.
$$

Define $\tilde{u}_{r i}$ as a new variable

$$
\tilde{u}_{r i}=\frac{x_{1 i}^{2} x_{4 i}^{2}}{x_{2 i}^{2}}-\frac{x_{1 i}}{x_{2 i}^{2}} u_{r i} .
$$

Substitute equation (9) into equation (8), and we have

$$
\left\{\begin{array}{l}
\dot{t}_{g i}=-1+\widetilde{u}_{r i}, \\
\dot{x}_{3 i}=x_{4 i}, \\
\dot{x}_{4 i}=-\frac{2 x_{2 i}}{x_{1 i}} x_{4 i}-\frac{u_{q i}}{x_{1 i}}+\frac{w_{q i}}{x_{1 i}} .
\end{array}\right.
$$

The research objectives of this paper are shown below. In the LOS direction, we design $u_{r i}$ to make sure that $t_{g i}$ can reach a consensus within a predefined time. $u_{q i}$ is proposed to ensure that $x_{3 i}$ and $x_{4 i}$ can approach zero within a predefined time.

2.2. Graph Theory. Assume $n$ missiles participate in the interception mission cooperatively. $G(N, I, W)$ is the graph of information communication among the multiple missiles. $G(N, I, W)$ is formed by node $N=\left\{N_{1}, N_{2}, \ldots, N_{n}\right\}$, $I \subseteq\left\{\left(N_{i}, N_{j}\right): N_{i}, N_{j} \in N\right\}$, and a weighted adjacency matrix $W=\left[c_{i j}\right] \in R_{n \times n}$. When the information is transferred from $N_{j}$ to $N_{i}$, the weight of edge $c_{i j}$ satisfies $c_{i j} \neq 0$. Otherwise, $c_{i j}=0$. It is worth noting that $G$ is undigraph if $\left(N_{i}, N_{j}\right) \in I \Leftrightarrow\left(N_{j}, N_{i}\right) \in I$. The adjacency matrix $W$ has symmetry in the undigraph $G$. If the undigraph $G$ is connected, there is a path connecting every two nodes [27]. The neighbor set of $N_{i}$ can be obtained as

$$
M_{i}=\left\{j \in N: c_{i j} \neq 0\right\}=\left\{j \in N:\left(N_{i}, N_{j}\right) \in I\right\}
$$

\subsection{Preliminaries}

Definition 1 (see [40-42]). Consider a nonlinear system:

$$
\dot{x}(t)=f(x(t), \vartheta), \quad x(0)=x_{0},
$$


where $x(t) \in R^{n}$ denotes the system state variable and $\vartheta \in R^{l}$ is the design parameter of system (12). System (12) is said to converge to the origin within a finite time if there exists a settling time function $T: R^{n} \longrightarrow R^{+}$, such that, for every $x_{0} \in R^{n}$, the solution of system (12) satisfies $\lim _{t \longrightarrow T\left(x_{0}\right)}$ $\Psi\left(t, x_{0}\right)=0$. For a globally finite-time convergent system (12), if for any initial state $x_{0} \in R^{n}$, there exists a bounded convergence time $T_{\max }$ irrelevant to initial conditions satisfying $T\left(x_{0}\right)<T_{\max }$, the origin has global fixed-time convergence.

Definition 2 (see [42-44]). The origin of the fixed-time stable system (12) is predefined-time stable if, for any convergence time $T_{c} \in R_{+}$, there is $\vartheta \in R^{l}$ such that the settling-time function of (12) satisfies $T\left(\mathbf{x}_{0}\right) \leq T_{c}$.

$$
\mathrm{x}_{0} \in \mathbb{R}^{n}
$$

Definition 3 (see [38]). Define a continuous function $\kappa: R_{+} \longrightarrow[0,1)$. It belongs to class $-\nabla$, and there is $\kappa \in \nabla_{1} . \kappa$ is monotonically increasing such that $\kappa(0)=0$ and $\lim _{r \rightarrow \infty} \kappa(r)=1$.

Notion 1 (see [38]). For a given vector $\mathbf{x}=\left[x_{1}\right.$, $\left.x_{2}, \ldots, x_{n}\right]^{T}$, it can be obtained that $\operatorname{sig}^{a}(x)=|x|^{a} \operatorname{sign}(x)$ and $\operatorname{sig}^{a}(\mathbf{x})=\left[\left|x_{1}\right|^{a} \operatorname{sign}\left(x_{1}\right),\left|x_{2}\right|^{a} \quad \operatorname{sign}\left(x_{2}\right), \ldots,\left|x_{n}\right|^{a}\right.$ $\left.\operatorname{sign}\left(x_{n}\right)\right]^{T}$, where $\operatorname{sign}(x)$ denotes the sign function.

Lemma 1 (see [38]). For system (12), define an unbounded Lyapunov function $V(x)$ and let $\kappa \in \nabla_{1}$ be differentiable in $R \backslash\{0\}$. For any predefined convergence time $T_{c} \in R_{+}$and $\vartheta\left(t, x_{0}\right) \in R^{l}$, the time-derivative of $V(x)$ satisfies

$$
\dot{V}(x) \leq-\frac{1}{(1-p) T_{c}} \frac{(\kappa(V))^{P}}{\kappa^{\prime}(V)}, \quad \text { for } x \in R^{n} \backslash\{0\},
$$

where $0<p<1$, such that system (12) is predefined-time stable.

Lemma 2. [45]. Define a Lyapunov function $V(x)$ and let $\kappa(V)=V^{\xi} / V^{\xi}+\chi$, with $\chi>0$, such that

$$
\dot{V} \leq-\frac{1}{\xi \chi(1-p) T_{c}} V^{\xi p-\xi+1}\left(V^{\xi}+\chi\right)^{2-p}+\Omega,
$$

for any solution $\vartheta\left(t, x_{0}\right)$ of system (12), where $T_{c}>0$ denotes a predefined-time parameter, $\chi>0$ is a tuning parameter to adjust the convergence speed, $\Omega>0$ is a small positive number, and $0<\xi<1$ stands for an exponential coefficient. Then, system (12) origin has global predefined-time stability, with predefined time $T_{c}$.

Proof. Firstly, system (14) can be transformed into a differential equation:

$$
\dot{V}=-\frac{1}{\xi \chi(1-p) T_{c}} V^{\xi p-\xi+1}\left(V^{\xi}+\chi\right)^{2-p} .
$$

By integrating equation (15), there is

$$
\begin{aligned}
T\left(x_{0}\right) & =-\int_{V\left(x_{0}\right)}^{0} \frac{\mathrm{d} V}{\left(1 / \xi \chi(1-p) T_{c}\right) V^{\xi p-\xi+1}\left(V^{\xi}+\Upsilon\right)^{2-p}} \\
& =\xi \chi(1-p) T_{c} \int_{0}^{V\left(x_{0}\right)} \frac{\mathrm{d} V}{V^{\xi p-\xi+1}\left(V^{\mu}+\chi\right)^{2-p}} \\
& =(1-p) T_{c} \int_{0}^{V\left(x_{0}\right)} \frac{\xi \chi V^{\xi-1} /\left(V^{\xi}+\chi\right)^{2}}{\left(V^{\xi} / V^{\xi}+\chi\right)^{p}} \mathrm{~d} V \\
& =\left.T_{c}\left\{\left(\frac{V^{\xi}}{V^{\xi}+\chi}\right)^{1-p}\right\}\right|_{0} ^{V\left(x_{0}\right)} \cdot \\
& =T_{c} \cdot\left(\frac{V^{\xi}\left(x_{0}\right)}{V^{\xi}\left(x_{0}\right)+\chi}\right)^{1-p}
\end{aligned}
$$

It is obvious that $\lim _{V\left(x_{0}\right) \rightarrow \infty}\left(V^{\xi}\left(x_{0}\right) / V^{\xi}\left(x_{0}\right)+\chi\right)=1$ such that $\lim _{V\left(\mathbf{x}_{0}\right) \longrightarrow \infty} T\left(x_{0}\right)=T_{c}$. Therefore, the upper bound of the settling time is $T_{c}$.

Theorem 1. Define a radially unbounded Lyapunov function $V(x)$ and let $\kappa(V)=(2 / \pi) \arctan (V)$ such that

$$
\dot{V} \leq-\left(\frac{2}{\pi}\right)^{p-1} \frac{\left(1+V^{2}\right)}{(1-p) T_{c}}(\arctan V)^{p}+\Omega,
$$

for any solution $9\left(t, x_{0}\right)$ of system (12), where $T_{c}>0$, $0<p<1$, and $\Omega>0$ is a small positive number. Then, the origin of system (12) has predefined-time stability, with predefined time $T_{c}$.

Proof. Firstly, system (17) can be transferred as a differential equation:

$$
\dot{V}=-\left(\frac{2}{\pi}\right)^{p-1} \frac{\left(1+V^{2}\right)}{(1-p) T_{c}}(\arctan V)^{p} .
$$

By integrating with equation (18), there is

$$
\begin{aligned}
T\left(x_{0}\right) & =-\int_{V\left(x_{0}\right)}^{0} \frac{\mathrm{d} V}{\left(1 /(1-p) T_{c}\right)\left(\pi\left(1+V^{2}\right)((2 / \pi) \arctan (V))^{p} / 2\right)} \\
& =\frac{2(1-p) T_{c}}{\pi} \int_{0}^{V\left(x_{0}\right)} \frac{\mathrm{d} V}{\left(1+V^{2}\right)((2 / \pi) \arctan (V))^{p}} \\
& =(1-p) T_{c} \int_{0}^{V\left(x_{0}\right)} \frac{2 / \pi\left(1+V^{2}\right)}{((2 / \pi) \arctan (V))^{p}} \mathrm{~d} V \\
& =\left.T_{c}\left\{\left(\frac{2}{\pi} \arctan (V)\right)^{1-p}\right\}\right|_{0} ^{V\left(x_{0}\right)} \\
& =T_{c} \cdot\left(\frac{2}{\pi} \arctan \left(V\left(x_{0}\right)\right)\right)^{1-p} .
\end{aligned}
$$


It is obvious that $\lim _{V\left(\mathbf{x}_{0}\right) \longrightarrow \infty} 2 / \pi \arctan \left(V\left(x_{0}\right)\right)=1$ such that $\lim _{V\left(\mathbf{x}_{0}\right) \rightarrow \infty} T\left(x_{0}\right)=T_{c}$. Therefore, the bound of the settling time is $T_{c}$. Then, the origin of system (12) has predefined-time stability, with predefined time $T_{c}$.

Lemma 3 (see [46]). For any $y_{i} \in R, i=1,2, \ldots, n$ $\left(\sum_{i=1}^{n}\left|y_{i}\right|\right)^{p} \leq \sum_{i=1}^{n}\left|y_{i}\right|^{p}$, where $p \in R^{+}$and $p \in(0,1]$.

Lemma 4 (see [46]). If $\eta \in R^{+}$and $\eta>1$, for any $x, y \in R$, there exists $|x+y|^{\eta} \leq 2^{\eta-1}\left|x^{\eta}+y^{\eta}\right|$.

\section{Predefined-Time Cooperative Guidance}

In this section, a novel predefined-time cooperative guidance law with impact angle constraints is designed, which consists of two parts: the cooperative guidance law $u_{r i}$ in the LOS direction and the cooperative guidance law $u_{q i}$ in the normal direction of LOS. And the strict proofs of the designed cooperative guidance law $u_{r i}$ and $u_{q i}$ are given. The structure diagram of the predefined-time cooperative guidance system is shown in Figure 2.

3.1. Guidance Law in the LOS Direction. From equation (5), the guidance system is formulated as

$$
\left\{\begin{array}{l}
\dot{x}_{1 i}=x_{2 i} \\
\dot{x}_{2 i}=x_{1 i} x_{4 i}^{2}-u_{r i}
\end{array}\right.
$$

The impact time $t_{F i}$ can be presented as

$$
t_{F i}=t+t_{g i} .
$$

Assume each missile can simultaneously arrive. $t_{g i}$ will achieve a consensus when $t_{F i}$ is convergent. $t_{F i}$ can be rewritten as

$$
\dot{t}_{F i}=\widetilde{u}_{r i}
$$

According to Definition 1, under the protocol $\widetilde{u}_{r i}, t_{F i}$ can uniformly converge within the predefined time.

Theorem 2. Suppose that the undigraph of system (12) is connected. There is a consensus protocol as shown in equation (23) which can make system (12) achieve predefined-time stability:

$$
\begin{aligned}
\tilde{u}_{r i}= & \left(\frac{2}{\pi}\right)^{\delta-1} \frac{\left(1+\left(1 / 4 \sum_{i=1}^{n} \sum_{j=1}^{n} a_{i j}\left(x_{j}-x_{i}\right)^{2}\right)^{2}\right)}{(1-\delta) T_{c 1}} \\
& \cdot \frac{\left(\arctan \left(1 / 4 \sum_{i=1}^{n} \sum_{j=1}^{n} a_{i j}\left(x_{j}-x_{i}\right)^{2}\right)\right)^{\delta}}{\left|\sum_{i=1}^{n} a_{i j}\left(x_{j}-x_{i}\right)\right|} \operatorname{sign} \\
& \left(\sum_{i=1}^{n} a_{i j}\left(x_{j}-x_{i}\right)\right),
\end{aligned}
$$

where $0<\delta<1$.
Proof. Consider the following Lyapunov candidate:

$$
V(x)=\frac{1}{4} \sum_{i=1}^{n} \sum_{j=1}^{n} a_{i j}\left(x_{j}-x_{i}\right)^{2} .
$$

Taking the derivative of $V(x)$ yields

$\dot{V}_{i}=\sum_{i=1}^{n} \frac{\partial V(x)}{\partial x_{i}} \dot{x}_{i}$

$$
\begin{aligned}
= & -\sum_{i=1}^{n} a_{i j}\left(x_{j}-x_{i}\right)\left(\frac{2}{\pi}\right)^{\delta-1} \frac{\left(1+\left(1 / 4 \sum_{i=1}^{n} \sum_{j=1}^{n} a_{i j}\left(x_{j}-x_{i}\right)^{2}\right)^{2}\right)}{(1-\delta) T_{c 1}} \\
& \times \frac{\left(\arctan \left(1 / 4 \sum_{i=1}^{n} \sum_{j=1}^{n} a_{i j}\left(x_{j}-x_{i}\right)^{2}\right)\right)^{\delta}}{\left|\sum_{i=1}^{n} a_{i j}\left(x_{j}-x_{i}\right)\right|} \text { sign } \\
& \left(\sum_{i=1}^{n} a_{i j}\left(x_{j}-x_{i}\right)\right) \\
= & -\left|\sum_{i=1}^{n} a_{i j}\left(x_{j}-x_{i}\right)\right|\left(\frac{2}{\pi}\right)^{\delta-1} \\
& \frac{\left(1+\left(1 / 4 \sum_{i=1}^{n} \sum_{j=1}^{n} a_{i j}\left(x_{j}-x_{i}\right)^{2}\right)^{2}\right)}{(1-\delta) T_{c 1}} \\
& \times \frac{\left(\arctan \left(1 / 4 \sum_{i=1}^{n} \sum_{j=1}^{n} a_{i j}\left(x_{j}-x_{i}\right)^{2}\right)\right)^{\delta}}{\left|\sum_{i=1}^{n} a_{i j}\left(x_{j}-x_{i}\right)\right|} \\
\leq & -\left(\frac{2}{\pi}\right)^{\delta-1} \frac{\left(1+\left(1 / 4 \sum_{i=1}^{n} \sum_{j=1}^{n} a_{i j}\left(x_{j}-x_{i}\right)^{2}\right)^{2}\right)}{(1-\delta) T_{c 1}} \\
& \times\left(\arctan \left(\frac{1}{4} \sum_{i=1}^{n} \sum_{j=1}^{n} a_{i j}\left(x_{j}-x_{i}\right)^{2}\right)\right)^{\delta} .
\end{aligned}
$$

Therefore, equation (24) can be transformed into

$$
\begin{gathered}
\dot{V}_{i} \leq-\left(\frac{2}{\pi}\right)^{\delta-1} \frac{\left(1+\left(1 / 4 \sum_{i=1}^{n} \sum_{j=1}^{n} a_{i j}\left(x_{j}-x_{i}\right)^{2}\right)^{2}\right)}{(1-\delta) T_{c 1}} \\
\cdot\left(\arctan \left(\frac{1}{4} \sum_{i=1}^{n} \sum_{j=1}^{n} a_{i j}\left(x_{j}-x_{i}\right)^{2}\right)\right)^{\delta} \\
\leq-\left(\frac{2}{\pi}\right)^{\delta-1} \frac{\left(1+V^{2}\right)}{(1-\delta) T_{c 1}} \cdot(\arctan (V))^{\delta} .
\end{gathered}
$$

According to Theorem 1, system (23) can achieve predefined-time stability by the proposed $\widetilde{u}_{r i}$ within the predefined time $T_{c 1}$, and this completes the proof of Theorem 2 . 


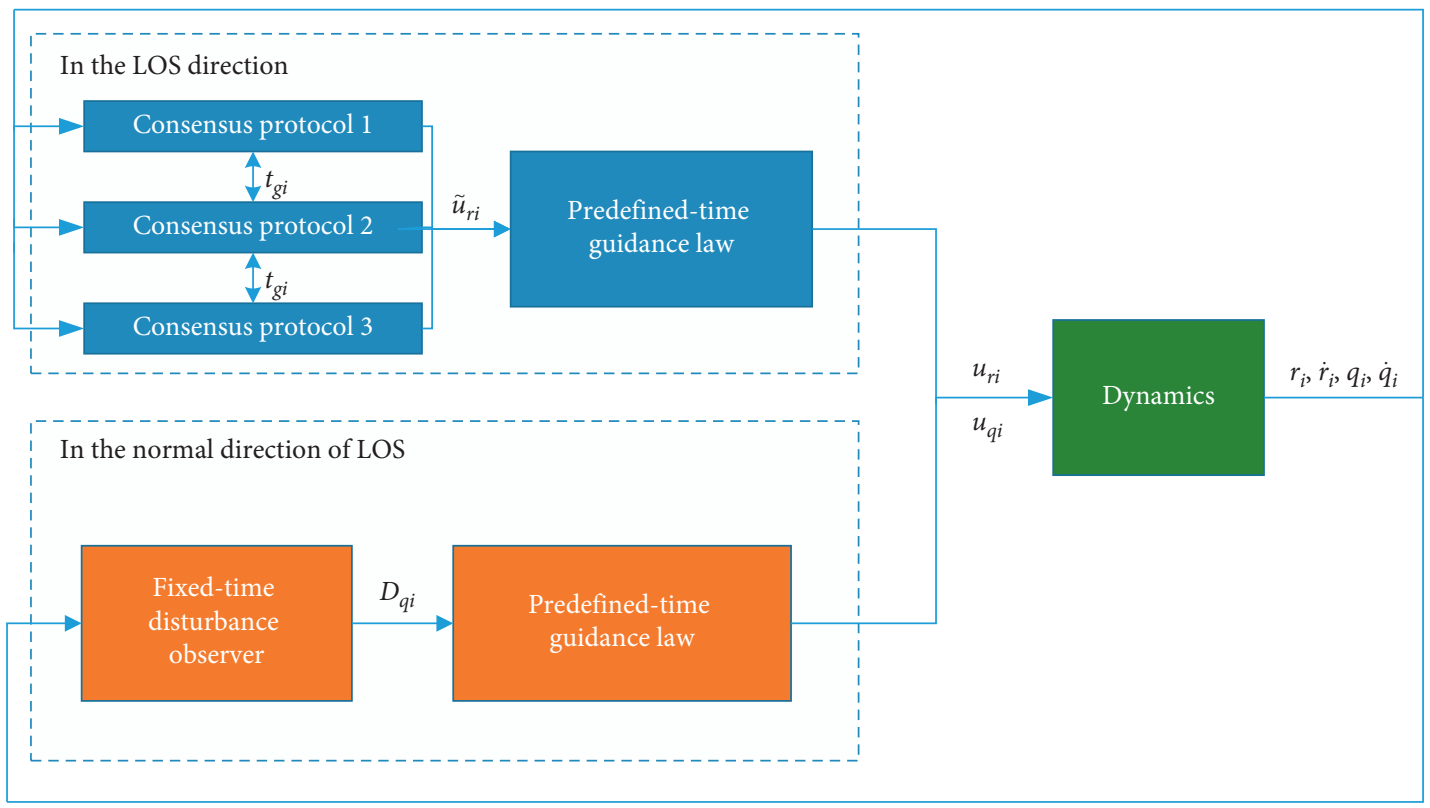

FIGURE 2: Structure diagram of the cooperative guidance system.

Theorem 3. Suppose that the undigraph is connected in the multiple-missile system. The guidance law $u_{r i}$ as shown in equation (27) can make $u_{r i}$ achieve the predefined-time consensus:

$$
u_{r i}=x_{1 i} x_{4 i}^{2}-\frac{x_{2 i}^{2}}{x_{1 i}} \widetilde{u}_{r i}
$$

where

$$
\begin{aligned}
\tilde{u}_{r i}= & \left(\frac{2}{\pi}\right)^{\delta-1} \frac{\left(1+\left(1 / 4 \sum_{i=1}^{n} \sum_{j=1}^{n} a_{i j}\left(\left(x_{1 i} / x_{2 i}\right)-\left(x_{1 j} / x_{2 j}\right)\right)^{2}\right)^{2}\right)}{(1-\delta) T_{c 1}} \\
& \cdot \frac{\left(\arctan \left(1 / 4 \sum_{i=1}^{n} \sum_{j=1}^{n} a_{i j}\left(a_{i j}\left(\left(x_{1 i} / x_{2 i}\right)-\left(x_{1 j} / x_{2 j}\right)\right)^{2}\right)^{2}\right)\right)^{\delta}}{\left|\sum_{i=1}^{n} a_{i j}\left(\left(x_{1 i} / x_{2 i}\right)-\left(x_{1 j} / x_{2 j}\right)\right)\right|} \operatorname{sign}\left(\sum_{i=1}^{n} a_{i j}\left(\frac{x_{1 i}}{x_{2 i}}-\frac{x_{1 j}}{x_{2 j}}\right)\right) .
\end{aligned}
$$

Proof. Combine the derivative of $t_{F i}$ with equation (5), and we have

$$
\begin{aligned}
\dot{t}_{F i} & =\frac{\dot{x}_{4 i}^{2}}{\dot{x}_{2 i}^{2}}-\frac{x_{1 i}}{\dot{x}_{2 i}^{2}} u_{r i} \\
& =\left(\frac{2}{\pi}\right)^{\delta-1} \frac{\left(1+\left(1 / 4 \sum_{i=1}^{n} \sum_{j=1}^{n} a_{i j}\left(t_{F j}-t_{F i}\right)^{2}\right)^{2}\right)}{(1-\delta) T_{c 1}} \\
& . \frac{\left(\arctan \left(1 / 4 \sum_{i=1}^{n} \sum_{j=1}^{n} a_{i j}\left(t_{F j}-t_{F i}\right)^{2}\right)\right)^{\delta}}{\left|\sum_{i=1}^{n} a_{i j}\left(t_{F j}-t_{F i}\right)\right|} \operatorname{sign}\left(\sum_{i=1}^{n} a_{i j}\left(t_{F j}-t_{F i}\right)\right) .
\end{aligned}
$$

According to Theorem 2, $t_{F i}$ can reach a consensus if $t>t_{F i}$. It demonstrates that each missile can exchange information in the guidance process and simultaneously intercept the target.
Remark 1. The proposed guidance law $u_{r i}$ ensures that $t_{F i}$ can reach a consensus within the predefined time $T_{c 1}$ depending on the consensus protocol in equation (23). The convergence time can be adjusted based on the predefined time $T_{c 1}$. In addition, by changing the magnitude of the design parameter $\delta$, the convergence speed can be further improved.

3.2. Fixed-Time Disturbance Observer. The cooperative guidance model of equation (5) in the normal direction of LOS can be presented as follows:

$$
\left\{\begin{array}{l}
\dot{x}_{3 i}=x_{4 i}, \\
\dot{x}_{4 i}=-\frac{2 x_{2 i}}{x_{1 i}} x_{4 i}-\frac{u_{q i}}{x_{1 i}}+D_{q i},
\end{array}\right.
$$

where $D_{q i}=w_{q i} / x_{1 i}$ denotes the disturbance caused by the target acceleration. 
However, the external disturbance $D_{q i}$ is unmeasurable during the flight. In order to solve the problem, an FxTDO is used for the estimation of the external disturbance $D_{q i}$. Then, the estimated disturbance can compensate for the system. The disturbance observer (see [47]) is defined as

$$
\left\{\begin{array}{l}
\dot{z}_{1}=z_{2}+\theta \varepsilon_{1} \rho\left(x_{2}-z_{1}, \eta_{1}, \eta_{2}\right)-\frac{2 x_{2 i}}{x_{1 i}} x_{4 i}-\frac{u_{q i}}{x_{1 i}}, \\
\dot{z}_{2}=\theta^{2} \varepsilon_{2} \rho\left(x_{2}-z_{1}, \eta_{1}, \eta_{2}\right),
\end{array}\right.
$$

where $z_{1}$ and $z_{2}$ are estimated values of $x_{2}$ and the disturbance $D_{q i}$, respectively. $\eta_{1}, \eta_{2}, \varphi, \varepsilon_{1}$, and $\varepsilon_{2}$ are the design parameters, satisfying $\varepsilon_{1}, \varepsilon_{2}>0, \quad \varepsilon_{1} \geq 2 \sqrt{\varepsilon_{2}}, \quad \varphi \geq 0$, $\eta_{1} \in(0.5,1)$, and $\eta_{2} \in(1,2) . \rho(\cdot)$ as the correction term is defined by

$$
\rho\left(x, \eta_{1}, \eta_{2}\right)=\left\{\begin{array}{l}
\operatorname{sig}^{\eta_{1}} x,|x|<1, \\
\operatorname{sig}^{\eta_{2}} x,|x| \geq 1 .
\end{array}\right.
$$

The stability proof of the above disturbance observer can be found in [47]. In conclusion, the observation errors of $\left|x_{2 i}-z_{1 i}\right|$ and $\left|D_{q i}-z_{2 i}\right|$ are fixed-time convergent to the origin, and the fixed time $T_{d i}$ satisfies

$$
T_{d i}(x) \leq \frac{4}{\varphi}\left(\frac{1}{1-\eta_{1}}+\frac{1}{\eta_{2}-1}\right) .
$$

Furthermore, the actual convergence time of the FxTDO is required to be smaller than the convergence time of the guidance system.

3.3. Guidance Law in the Normal Direction of LOS. A new predefined-time guidance law in the normal direction of LOS is proposed to ensure that each missile can achieve the desired LOS angle. Then, a new sliding mode surface is presented as follows:

$$
s_{i}=x_{4 i}+\gamma_{0} \operatorname{sig}^{\mu p_{1}-\mu+1}\left(x_{3 i}\right)\left(\left|x_{3 i}\right|^{\mu}+\Upsilon\right)^{2-p_{1}},
$$

where $\gamma_{0}=1 /\left(\mu \Upsilon\left(1-p_{1}\right) T_{c 2}\right), 1<\Upsilon<2,0.5<\mu<1, T_{c 2}>0$, and $0<p_{1}<1$. Therefore, we can obtain the following results.

Theorem 4. For the cooperative guidance model in (30), the sliding mode surface can be designed as (34). Then, $x_{3 i}=0$ and $x_{4 i}=0$ can be achieved within the predefined time $T_{c 2}$.

Proof. Suppose the controller can guarantee $s_{i}=0$ regardless of external disturbances. Then, we have

$$
\begin{aligned}
& x_{4 i}+\gamma_{0} \operatorname{sig}^{\mu p_{1}-\mu+1}\left(x_{3 i}\right)\left(\left|x_{3 i}\right|^{\mu}+\Upsilon\right)^{2-p_{1}}=0, \\
& x_{4 i}=-\gamma_{0} \operatorname{sig}^{\mu p_{1}-\mu+1}\left(x_{3 i}\right)\left(\left|x_{3 i}\right|^{\mu}+\Upsilon\right)^{2-p_{1}} .
\end{aligned}
$$

Consider a Lyapunov function candidate as $V_{1 i}=\left|x_{3 i}\right|$, and the time-derivative of $V_{1 i}$ is

$$
\begin{aligned}
\dot{V}_{1 i} & =\dot{x}_{3 i} \operatorname{sign}\left(x_{3 i}\right) \\
& =-\gamma_{0}\left|x_{3 i}\right|^{\mu p_{1}-\mu+1}\left(\left|x_{3 i}\right|^{\mu}+\Upsilon\right)^{2-p_{1}} \\
& =-\frac{1}{\mu \Upsilon\left(1-p_{1}\right) T_{c 2}} V_{1 i}^{\mu p_{1}-\mu+1}\left(V_{1 i}^{\mu}+\Upsilon\right)^{2-p_{1}} .
\end{aligned}
$$

Based on Lemma 1, the proposed sliding mode surface is predefined-time stable. $x_{3 i} \longrightarrow 0$ and $x_{4 i} \longrightarrow 0$ are obtained in the predefined time $T_{c 2}$. This completes the proof of Theorem 4.

With the sliding mode surface in equation (34), the predefined-time cooperative guidance law can be designed as

$$
u_{i}=\frac{1}{x_{1 i}}\left\{\begin{array}{c}
\frac{1}{\left(1-p_{2}\right) T_{c 3}}\left(\frac{2}{\pi}\right)^{p_{2}-1}\left(\arctan \left(\left|s_{i}\right|\right)\right)^{p_{2}}\left(1+s_{i}^{2}\right) \operatorname{sign}\left(s_{i}\right) \\
-\frac{2 x_{2 i}}{x_{1 i}} x_{4 i}+z_{2 i}+F_{i}
\end{array}\right\} \text {, }
$$

where $z_{2 i}$ is the output signal of the FxTDO. $F_{i}$ represents the term related to the derivative of $s_{i}$. Then, we have

$$
\dot{s}=\dot{x}_{4 i}+x_{4 i}\left\{\begin{array}{c}
\gamma_{0}\left|x_{3 i}\right|^{\mu p_{1}-\mu}\left(\left|x_{3 i}\right|^{\mu}+\Upsilon\right)^{2-p_{1}}+\gamma_{0} \operatorname{sig}^{\mu p_{1}-\mu+1}\left(x_{3 i}\right)\left(2-p_{1}\right) \\
\left(\left|x_{3 i}\right|^{\mu}+\Upsilon\right)^{1-p_{1}} \mu \operatorname{sig}^{\mu-1}\left(x_{3 i}\right)
\end{array}\right\},
$$

where

$$
F_{i}=x_{4 i}\left\{\begin{array}{c}
\gamma_{0}\left|x_{3 i}\right|^{\mu p_{1}-\mu}\left(\left|x_{3 i}\right|^{\mu}+\Upsilon\right)^{2-p_{1}} \\
+\operatorname{sig}^{\mu p_{1}-\mu+1}\left(x_{3 i}\right)\left(2-p_{1}\right)\left(\left|x_{3 i}\right|^{\mu}+\Upsilon\right)^{1-p_{1}} \mu\left|x_{3 i}\right|^{\mu-1}
\end{array}\right\} .
$$

Theorem 5. If the PTCG law is designed as (37), then the sliding mode surface $s_{i}=0$ can be achieved within the predefined time.

Proof. Substituting equation (30) into $\dot{s}_{i}$ yields

$$
\dot{s}_{i}=a_{i}+b_{i} u_{q i}+D_{q i}+F_{i},
$$

where $a_{i}=-\left(2 x_{2 i} x_{4 i} / x_{1 i}\right)$ and $b_{i}=-1 / x_{1 i}$.

Bring equation (37) into equation (40), and we have

$$
\begin{aligned}
\dot{s}_{i}= & a_{i}+b_{i} u_{q i}+D_{q i}+F_{i} \\
= & -\left\{\left(\frac{2}{\pi}\right)^{p_{2}-1} \frac{\left(\arctan \left(\left|s_{i}\right|\right)\right)^{p_{2}}\left(1+s_{i}^{2}\right) \operatorname{sign}\left(s_{i}\right)}{\left(1-p_{2}\right) T_{c 3}}+a_{i}+z_{2 i}+F_{i}\right\}, \\
& +a_{i}+D_{q i}+F_{i} \\
= & -\left(\frac{2}{\pi}\right)^{p_{2}-1} \frac{\left(\arctan \left(\left|s_{i}\right|\right)\right)^{p_{2}}\left(1+s_{i}^{2}\right) \operatorname{sign}\left(s_{i}\right)}{\left(1-p_{2}\right) T_{c 3}}-z_{2 i}+D_{q i} .
\end{aligned}
$$




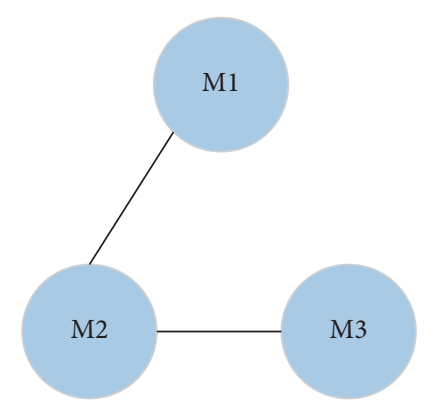

FIgURE 3: Communication topology among the missiles.

Considering the Lyapunov function,

$$
V_{2 i}=\left|s_{i}\right|
$$

Taking the derivative of $V_{2 i}$ yields

$$
\begin{aligned}
\dot{V}_{2 i} & =\dot{s}_{i} \operatorname{sign}\left(\dot{s}_{i}\right) \\
& =-\frac{2^{p_{2}-1}}{\pi^{p_{2}-1}} \frac{\left(1+\left|s_{i}\right|^{2}\right)}{\left(1-p_{2}\right) T_{c 3}}\left(\arctan \left(\left|s_{i}\right|\right)\right)^{p_{2}}+\left(D_{i}-z_{2 i}\right) \operatorname{sign}\left(s_{i}\right) \\
& =-\frac{2^{p_{2}-1}}{\pi^{p_{2}-1}} \frac{\left(1+V_{2 i}^{2}\right)}{\left(1-p_{2}\right) T_{c 3}}\left(\arctan \left(V_{2 i}\right)\right)^{p_{2}}+\left(D_{i}-z_{2 i}\right) \operatorname{sign}\left(V_{2 i}\right) .
\end{aligned}
$$

For the disturbance observer in (31), the observation error $\left|D_{q i}-z_{2 i}\right|$ will approach zero in a fixed time. Thus, there exists a convergence time $T_{d r i}$, satisfying $T_{d r i}<T_{c 3}$, and we can get

$$
\left|D_{q i}-z_{2 i}\right| \leq N, \quad t>T_{d r i}
$$

where $N$ is a small constant.

Then, (43) can be simplified as

$$
\begin{aligned}
\dot{V}_{2 i} & \leq-\frac{2^{p_{2}-1}}{\pi^{p_{2}-1}} \frac{\left(1+\left|s_{i}\right|^{2}\right)}{\left(1-p_{2}\right) T_{c 3}}\left(\arctan \left(\left|s_{i}\right|\right)\right)^{p_{2}}+N \\
& \leq-\frac{2^{p_{2}-1}}{\pi^{p_{2}-1}} \frac{\left(1+V_{2 i}^{2}\right)}{\left(1-p_{2}\right) T_{c 3}}\left(\arctan \left(V_{2 i}\right)\right)^{p_{2}}+\Omega .
\end{aligned}
$$

By utilizing Theorem 1 , the sliding mode surface $s=0$ can be reached within the predefined time $T_{c 3}$. This finishes the proof of Theorem 5, which means that the design of the PTCG law for the multimissile system is completed. Consequently, the PTCG law can achieve predefined-time stability with the proposed $u_{i}$ in the predefined-time $T_{c}=T_{c 2}+T_{c 3}$.

Remark 2. By employing the FxTDO, the disturbance information can be obtained, which enhances the guidance accuracy of the given PTCG law. In fact, considering the convergence time of the observer can affect the convergence time of the whole system, the predefined convergence time of the guidance system should be $T_{c}=T_{c 2}+T_{c 3}+T_{d r i}$ in the existence of uncertainties. And the system reaches the sliding mode surface $s=0$ within the predefined-time interval $T_{c 3}+T_{d r i}$. However, $T_{d r i}$ can be covered by $T_{c 3}$ when the predefined time $T_{c 3}$ is larger than the convergence time from the initial state of the system to the sliding surface according to Theorem 5. Furthermore, it is necessary to select an appropriate $T_{c 2}$ or $T_{c 3}$ as the upper bound. The adjustments of the guidance law parameters $T_{c 2}, T_{c 3}, p_{1}$, and $p_{2}$ are based on the simulation results.

\section{Simulations and Results}

To verify the effectiveness of the given PTCG, the case where three missiles intercept a moving target is simulated. The initial values of the target are set as $V_{T}=300 \mathrm{~m} / \mathrm{s}$ and $a_{T}=45 \cos (t) \mathrm{m} / \mathrm{s}^{2}$. The communication topology of the missiles is shown in Figure 3, and the weighted adjacency matrix of the communication topology is $A=[010 ; 101 ; 010]$. The lateral acceleration of three interceptors satisfies $a_{i \max }=200 \mathrm{~m} / \mathrm{s}^{2}$. The initial values of this scenario are given in Table 1.

The parameters of the proposed PTCG law and FxTDO are given in Table 2.

Simulation results of the comparative experiment by the proposed PTCG law and by the finite-time cooperative guidance law in [27] in the same circumstance are presented in Figures 4-11 and Table 3.

From Figure 4 and Table 3, it is demonstrated that both PTCG and FTCG can intercept the maneuvering target simultaneously. Furthermore, the proposed PTCG has higher precision and shorter interception time than the FTCG in the mission.

As shown in Figure 5, the LOS angles $q_{i}$ can converge to the desired values by these two cooperative guidance laws. And all, the LOS rates can approach zero as shown in Figure 6. More importantly, Figures 5 and 6 also show that the convergence times of $q_{i}$ and $q_{d i}$ are both less than $5 \mathrm{~s}$, and the convergence curves are smoother under the PTCG. Meanwhile, it is observed that the given PTCG can ensure that the guidance system achieves predefined-time stability within $9.5 \mathrm{~s}$. In terms of the convergence rate, PTCG converges faster than FTCG.

As illustrated in Figure 7, the sliding mode surface of the PTCG achieves predefined-time stability within $4.5 \mathrm{~s}$. Hence, the predefined-time stability of the given PTCG law is verified by the above results, and the proposed PTCG performs better than the FTCG. Although the sliding dynamics is broken in the terminal flight phase, that is, the absolute value of the sliding mode surface does not equal 
TABLE 1: Initial values of the missiles and target.

\begin{tabular}{lcccc}
\hline Missile & Initial position $(\mathrm{m}, \mathrm{m})$ & Heading angle $\left(^{\circ}\right)$ & Desired angle $\left(^{\circ}\right)$ & Initial velocity $(\mathrm{m} / \mathrm{s})$ \\
\hline M1 & $(5940,-1040)$ & 11 & -6 & 610 \\
M2 & $(4900,-850)$ & -10 & 0 & 610 \\
M3 & $(4105,995)$ & -10 & 5 & 600 \\
Target & $(0,0)$ & 60 & - & 300 \\
\hline
\end{tabular}

TABle 2: Parameters of the PTCG.

\begin{tabular}{|c|c|c|c|c|c|c|c|c|c|c|c|c|c|}
\hline$T_{c}$ & $T_{c 1}$ & $\delta$ & $p_{1}$ & $\mu$ & $\gamma$ & $T_{c 2}$ & $p_{2}$ & $T_{c 3}$ & $\eta_{1}$ & $\eta_{2}$ & $\varepsilon_{1}$ & $\varepsilon_{2}$ & $\theta$ \\
\hline 9.5 & 1.5 & 0.95 & 0.8 & 0.8 & 1.6 & 4.5 & 0.4 & 5 & 0.7 & 1.5 & 10 & 20 & 3 \\
\hline
\end{tabular}

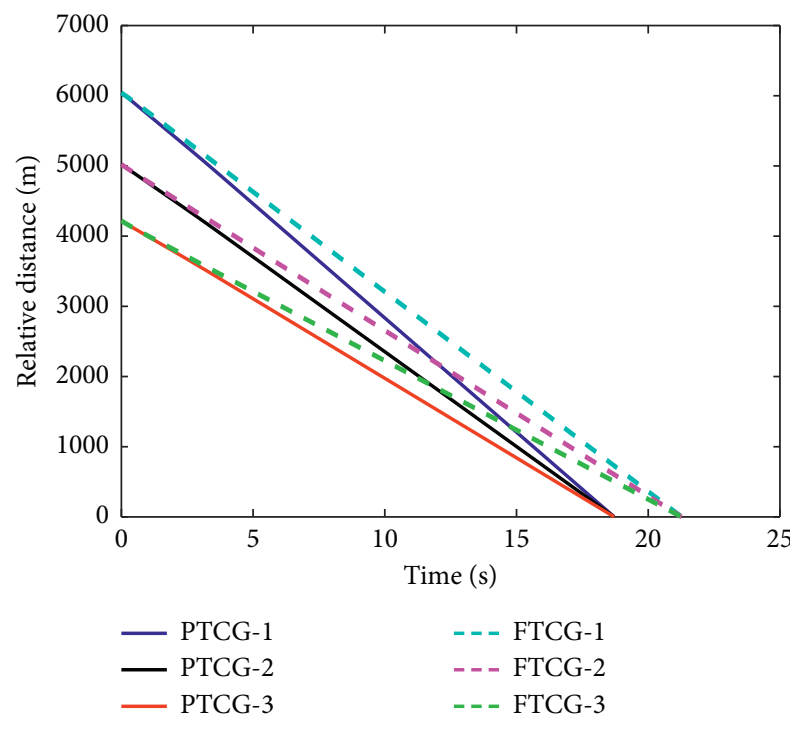

Figure 4: Curves of relative distance.

TABLE 3: Final parameters.

\begin{tabular}{ccccc}
\hline & Missile & M1 & M2 & M3 \\
\hline \multirow{4}{*}{ PTCG } & Miss distance (m) & 0.05 & 0.09 & 0.16 \\
& Impact LOS angle $\left({ }^{\circ}\right)$ & 5.001 & 0.001 & -6.001 \\
& Interception time (s) & 18.71 & 18.71 & 18.71 \\
\hline \multirow{3}{*}{ FTCG } & Miss distance (m) & 0.34 & 0.47 & 0.45 \\
& Impact LOS angle ( $\left.{ }^{\circ}\right)$ & 5.19 & 0.17 & -6.13 \\
& Interception time (s) & 21.26 & 21.26 & 21.26 \\
\hline
\end{tabular}

zero, it owns to the finite sampling frequency of the mathematical simulation. When the relative distance between missile and target turns to a small amount, the LOS angle velocity becomes large, which is indicated from equation (30), and the absolute value of the sliding mode variable increases.

In Figure 8, a relatively large acceleration command $u_{q i}$ for the three missiles was given in the initial phase of flight, since a larger acceleration command can drive $\dot{q}$ to zero at a

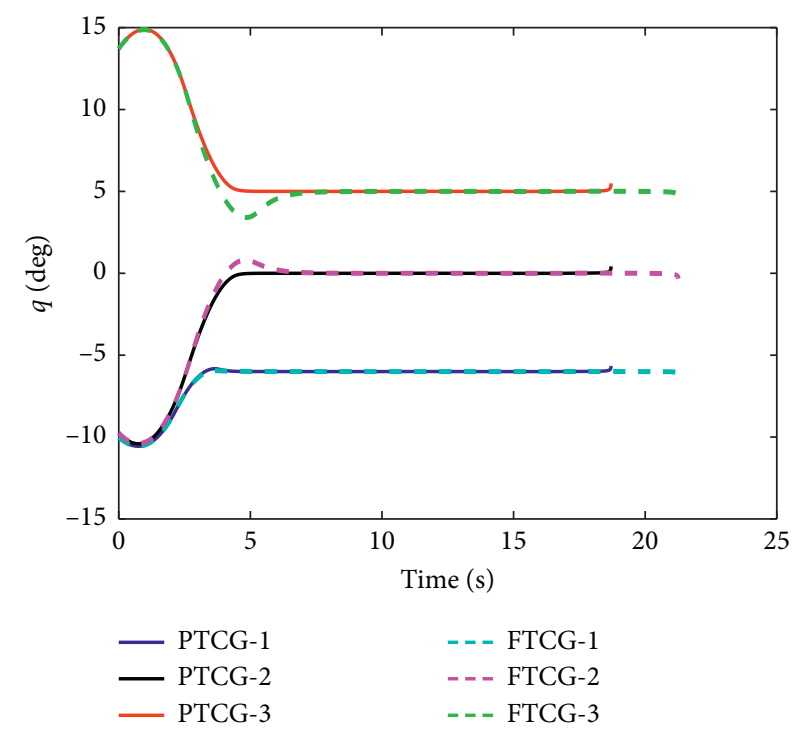

FIgURE 5: Curves of LOS angle.

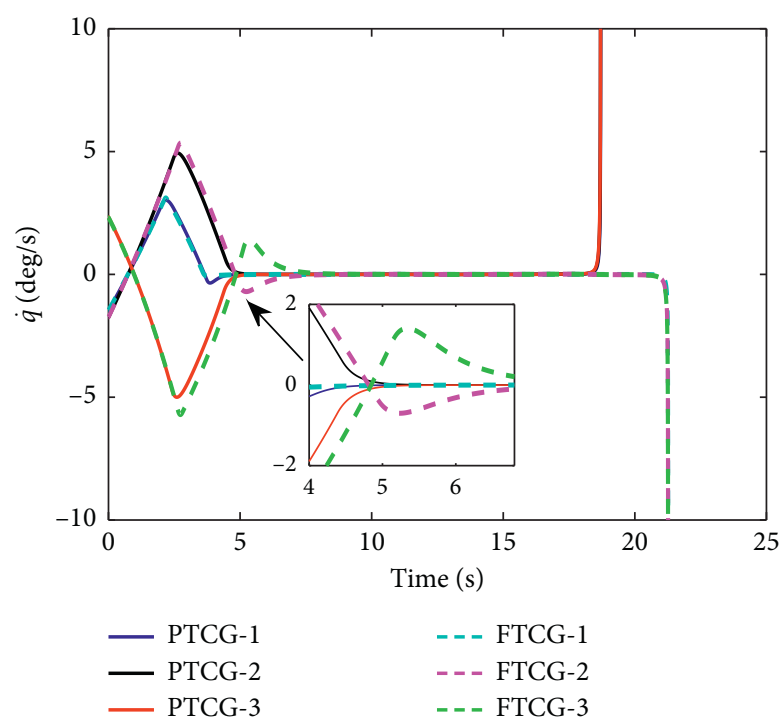

FIgURE 6: Curves of LOS angular velocity. 


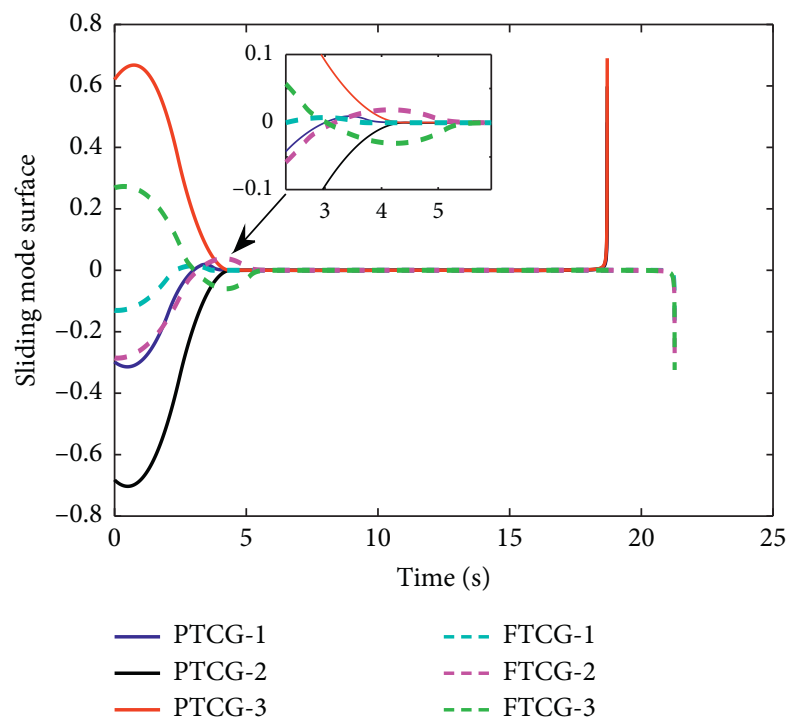

FIgURE: Curves of sliding mode surface.

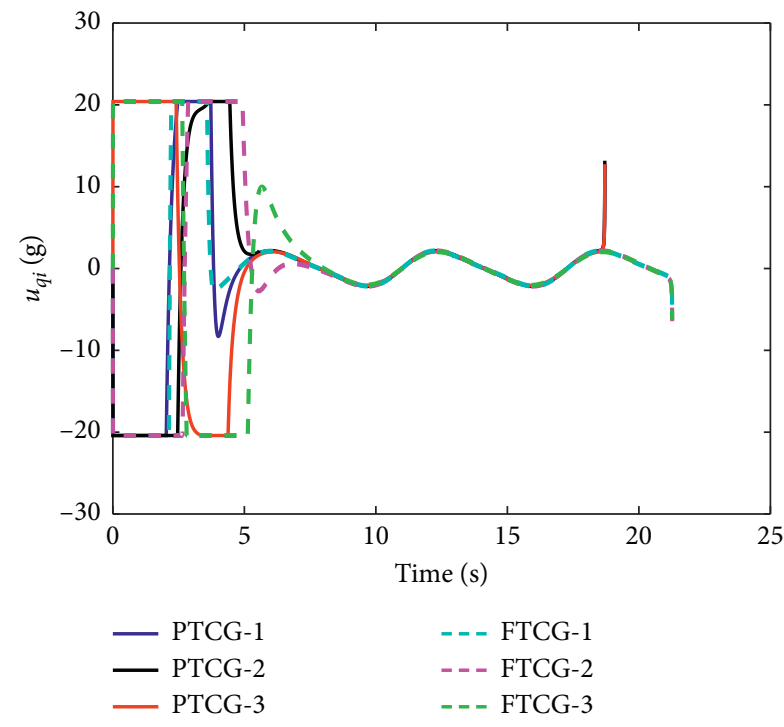

FIgURE 8: Acceleration command $u_{q i}$.

faster rate so that $q$ can converge to the desired value more quickly. As shown in Figure 9, by using the PTCG, it takes around $0.4 \mathrm{~s}$ for $t_{g i}$ to reach a consensus, which is faster than using the FTCG. Furthermore, Figure 9 demonstrates that the PTCG can achieve predefined-time stability within $1.5 \mathrm{~s}$.

The acceleration command $u_{r i}$ is shown in Figure 10. $u_{r i}$ is utilized to regulate $t_{g i}$ of the multiple missiles so that $t_{g i}$ realizes uniform convergence within a predefined time. $u_{r i}$ is

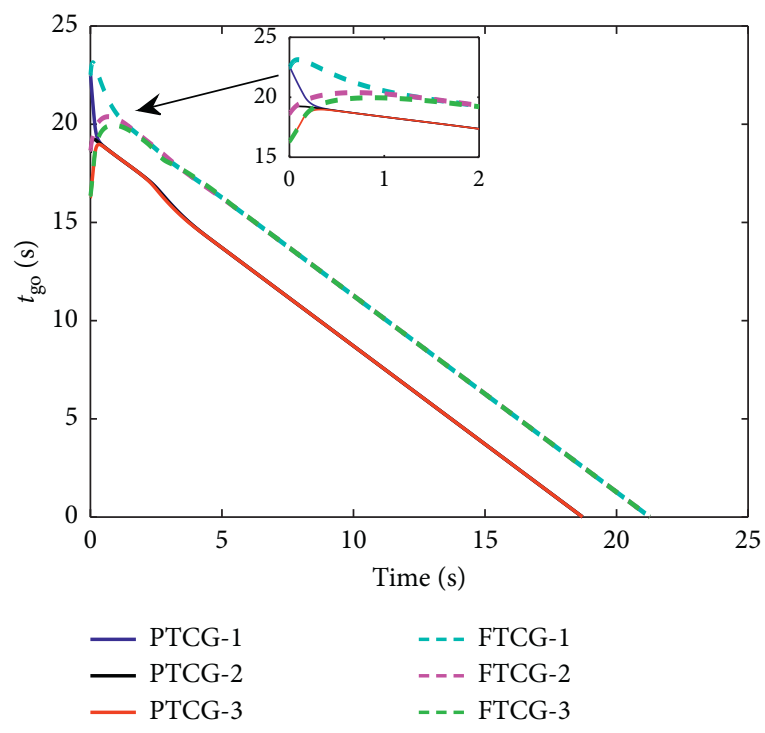

Figure 9: Time-to-go for three missiles.

quite large at the beginning, which reflects the fact that the less $t_{g i}$ it takes for the system to reach a consensus, the more energy it needs. In Figure 11, it is observed that the external disturbance is estimated accurately by the FxTDO, and all convergence time $T_{d r i}$ is less than $0.2 \mathrm{~s}$. In conclusion, the proposed PTCG law enjoys predefined-time convergence compared with the FTCG law. Hence, the guidance system has a faster convergence rate and higher precision by the proposed PTCG. 


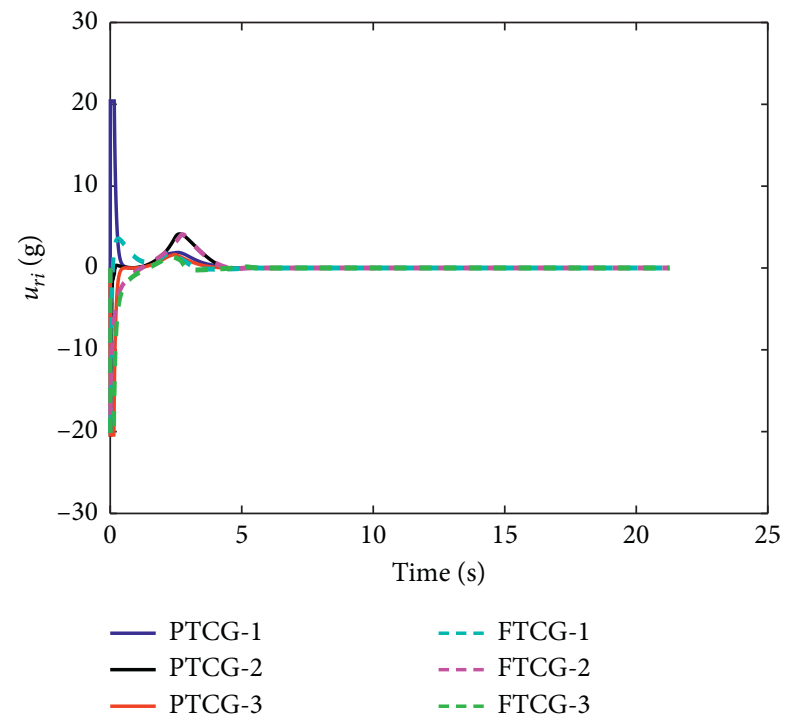

Figure 10: Acceleration command $u_{r i}$.
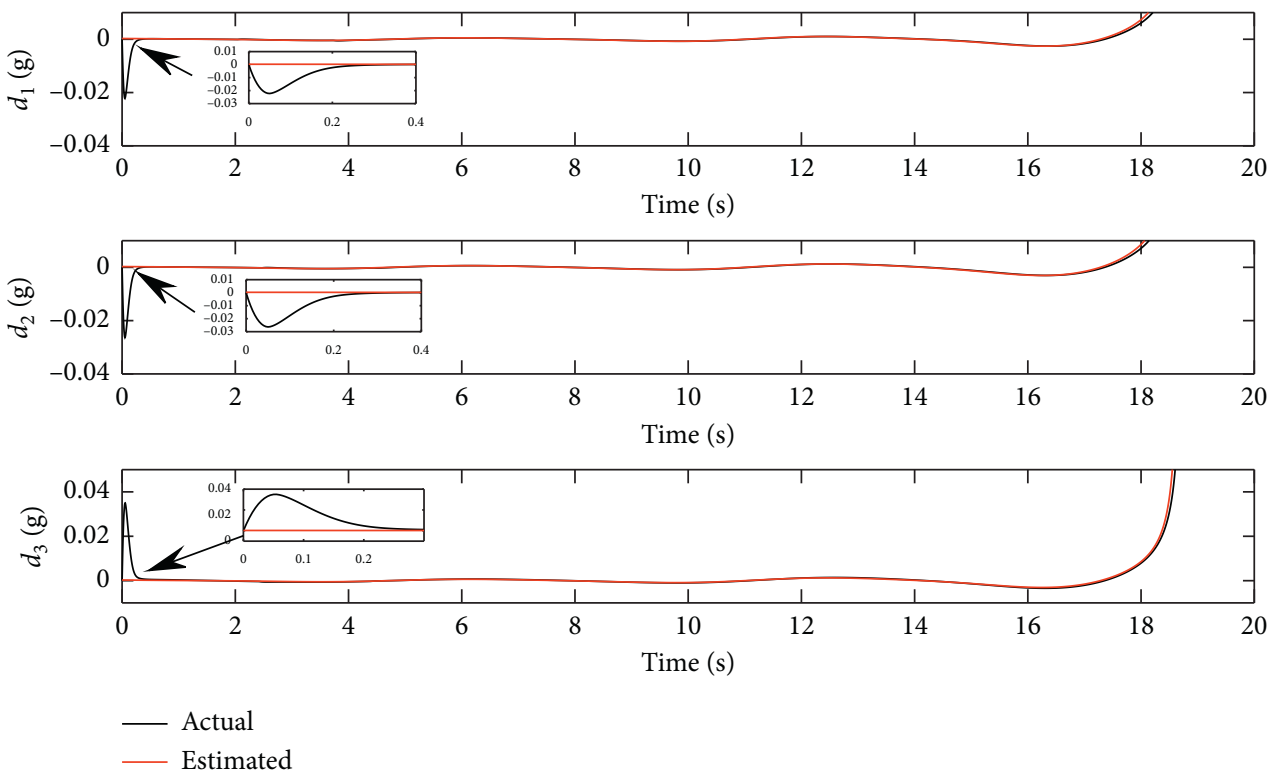

FIgURE 11: Disturbance estimation results.

\section{Conclusion}

In this paper, multiple missiles intercepting a maneuvering target have been solved by the proposed PTCG law. Under a novel predefined-time consensus protocol, a PTCG law along the LOS direction is designed. The designed PTCG law enables the time-to-go of the multiple missiles to reach a consensus in the predefined time. Considering the disturbance caused by the target maneuver, a PTCG law with an FxTDO in the normal direction of the LOS direction is presented. Thus, the guidance system can guarantee predefined-time stability. Meanwhile, strict proof of predefinedtime stability for the proposed PTCG law is presented. Finally, the designed PTCG law was validated by numerical simulations, which indicates the strong robustness and adaptability of the proposed PTCG. In the future work, we will further improve the proposed algorithm by considering switching communication topologies and interception of hypersonic vehicles in the design of the cooperative guidance law.

\section{Data Availability}

The data used to support the findings of this study are available from the corresponding author upon request.

\section{Conflicts of Interest}

The authors declare that they have no conflicts of interest. 


\section{Acknowledgments}

This study was supported by the Open Fund of National Defence Key Discipline Laboratory of Micro-Spacecraft Technology (Grant no. HIT.KLOF.MST.2018028).

\section{References}

[1] B. Jung and Y. Kim, "Guidance laws for anti-ship missiles using impact angle and impact time," in Proceedings of the AIAA Guidance, Navigation, and Control Conference, pp. 3048-3060, AIAA Press, Keystone, CL, USA, August 2006.

[2] S. Y. Zhao and R. Zhou, "Cooperative guidance for multimissile salvo attack," Chinese Journal of Aeronautics, vol. 21, no. 6, pp. 533-539, 2008, Chinese.

[3] P. Zhang, H. H. T. Liu, X. Li, and Y. Yao, "Fault tolerance of cooperative interception using multiple flight vehicles," Journal of the Franklin Institute, vol. 350, no. 9, pp. 23732395, 2013.

[4] I. S. Jeon, J. I. Lee, and M. J. Tahk, "Impact-time-control guidance law for anti-ship missiles," IEEE Transactions on Control Systems Technology, vol. 14, no. 6, pp. 260-266, 2006.

[5] I. S. Jeon, J. I. Lee, and M. J. Tahk, "Guidance law to control impact time and angle," IEEE Transactions on Aerospace and Electronic Systems, vol. 43, no. 1, pp. 301-310, 2007.

[6] S. R. Kumar and D. Ghose, "Impact time guidance for large heading errors using sliding mode control," IEEE Transactions on Aerospace and Electronic Systems, vol. 51, no. 4, pp. 3123-3138, 2015.

[7] D. Cho, H. J. Kim, and M.-J. Tahk, "Nonsingular sliding mode guidance for impact time control," Journal of Guidance, Control, and Dynamics, vol. 39, no. 1, pp. 61-68, 2016.

[8] Y. Zhang, X. Wang, and G. Ma, "Impact time control guidance law with large impact angle constraint," Proceedings of the Institution of Mechanical Engineers, Part G: Journal of Aerospace Engineering, vol. 229, no. 11, pp. 2119-2131, 2015.

[9] Y. Zhang, X. Wang, and H. Wu, "Impact time control guidance law with field of view constraint," Aerospace Science and Technology, vol. 39, pp. 361-369, 2014.

[10] N. Harl and S. N. Balakrishnan, "Impact time and angle guidance with sliding mode control," IEEE Transactions on Control Systems Technology, vol. 20, no. 6, pp. 1436-1449, 2011.

[11] Y. P. Sun, W. P. Lin, and Z. E. Fan, "Study on optimal guidance law under multiple-constrained condition," Ordnance Industry Automation, vol. 32, no. 12, pp. 4-7, 2013, [Chinese].

[12] Y. G. Zhang and Y. A. Zhang, "Research on cooperative guidance for multi-missile based on bi-arcs," Journal of Naval Aeronautical and Astronautical University, vol. 24, no. 5, pp. 537-542, 2009, [Chinese].

[13] S. R. Kumar and D. Ghose, "Sliding mode control based guidance law with impact time constraints," in Proceedings of ACC, pp. 5760-5765, AIAA Press, Washington, D.C., USA, June 2013.

[14] X. Wang, Y. Zheng, and H. Lin, "Integrated guidance and control law for cooperative attack of multiple missiles," Aerospace Science and Technology, vol. 42, pp. 1-11, 2015.

[15] E. J. Zhao, "Multiple missiles cooperative guidance based on leader-follower strategy," in Proceedings of CGNCC, pp. 1163-1167, AIAA Press, Yantai, China, August 2014.

[16] T. M. Yu, Y. Ming, and Z. Rui, "Decentralized cooperative guidance for leader-follower multi-missile systems,"
Navigation Positioning and Timing, vol. 3, no. 2, pp. 20-24, 2017, [Chinese].

[17] S. Y. Zhao, "Design of time-constrained guidance laws via virtual leader approach," Chinese Journal of Aeronautics, vol. 23, no. 1, pp. 103-108, 2010.

[18] Q. Zhao, X. Dong, Z. Liang, C. Bai, J. Chen, and Z. Ren, "Distributed cooperative guidance for multiple missiles with fixed and switching communication topologies," Chinese Journal of Aeronautics, vol. 30, no. 4, pp. 1570-1581, 2017.

[19] J. Zhou and J. Yang, "Distributed guidance law design for cooperative simultaneous attacks with multiple missiles," Journal of Guidance, Control, and Dynamics, vol. 39, no. 10, pp. 2439-2447, 2016.

[20] Z. Jiang and R. Zhou, "Distributed three-dimensional cooperative guidance via receding horizon control," Chinese Journal of Aeronautics, vol. 29, no. 4, pp. 972-983, 2016.

[21] X. Liu, J. Cao, and C. Xie, "Finite-time and fixed-time bipartite consensus of multi-agent systems under a unified discontinuous control protocol," Journal of the Franklin Institute, vol. 356, no. 2, pp. 734-751, 2017.

[22] P. Tong, S. Chen, and L. Wang, "Finite-time consensus of multi-agent systems with continuous time-varying interaction topology," Neurocomputing, vol. 284, no. 9, pp. 187-193, 2018.

[23] Z. Zuo and L. Tie, "Distributed robust finite-time nonlinear consensus protocols for multi-agent systems," International Journal of Systems Science, vol. 47, no. 6, pp. 1366-1375, 2016.

[24] T. Lv, Y. Y. Lv, and C. J. Li, "Finite time cooperative guidance law for multiple missiles with line-of-sight angle constraint," Acta Armamentarii, vol. 39, no. 2, pp. 305-314, 2018, [Chinese].

[25] D. L. Hou, "Finite-time cooperative guidance laws for multiple missiles with acceleration saturation constraints," IET Control Theory \& Applications, vol. 9, no. 10, pp. 1525-1535, 2018.

[26] C. Guo and X.-G. Liang, "Cooperative guidance law for multiple near space interceptors with impact time control," International Journal of Aeronautical and Space Sciences, vol. 15, no. 3, pp. 281-292, 2014.

[27] J. H. Song, S. M. Song, and S. L. Xu, "A cooperative guidance law for multiple missiles to intercept maneuvering target," Journal of Astronautics, vol. 37, no. 12, pp. 1432-1440, 2016, [Chinese].

[28] J. Song, S. Song, and S. Xu, “Three-dimensional cooperative guidance law for multiple missiles with finite-time convergence," Aerospace Science and Technology, vol. 67, pp. 193205, 2017.

[29] G. Y. Li, "Cooperative guidance law with angle constraint to intercept maneuvering target," Journal of Systems Engineering and Electronics, vol. 41, no. 3, pp. 626-635, 2019, [Chinese].

[30] M. J. Zhang, J. J. Ma, and Y. Han, "Fixed-time cooperative guidance law for multiple missiles against maneuvering target," in Proceedings of CAC, pp. 3848-3853, IEEE Press, Xian, China, November 2018.

[31] M. J. Zhang and J. J. Ma, "Adaptive fixed-time cooperative intercept guidance law with line-of-sight angle constraint," in Proceedings of ICMA, pp. 1992-1998, IEEE Press, Tianjin, China, August 2019.

[32] L. Jing, L. Zhang, J. Guo, and N. Cui, "Fixed-time cooperative guidance law with angle constraint for multiple missiles against maneuvering target," IEEE Access, vol. 8, pp. 7326873277, 2020.

[33] Z. Y. Chen, W. C. Chen, X. M. Liu et al., “Three-dimensional fixed-time robust cooperative guidance law for simultaneous 
attack with impact angle constraint," Aerospace Science and Technology, vol. 110, pp. 1-16, 2021.

[34] M. Lin, X. Ding, C. Wang, L. Liang, and J. Wang, "Threedimensional fixed-time cooperative guidance law with impact angle constraint and prespecified impact time," IEEE Access, vol. 9, pp. 29755-29763, 2021.

[35] D. S. Juan, E. N. Sanchez, and A. G. Loukianov, "Predefinedtime stability of dynamical systems with sliding modes," in Proceedings of American Control Conference (ACC), pp. 5842-5846, IEEE Press, Chicago, IL, USA, July 2015.

[36] A. G. Loukianov, D. S. Juan, G. Gomez et al., "A class of predefined-time stable dynamical systems," IMA Journal of Mathematical Control and Information, vol. 35, no. Suppl 1, pp. 1-29, 2018.

[37] D. S. Juan, M. Defoort, and A. J. Muñoz, "A second order sliding mode controller with predefined time convergence," in Proceedings of 2018 15th International Conference on Electrical Engineering, Computing Science and Automatic Control (CCE), pp. 5842-5846, IEEE Press, Mexico City, Mexico, September 2018.

[38] E. R. Jiménez, A. J. Muñoz, and J. D. Sanchez, "A lyapunovlike characterization of predefined-time stability," IEEE Transactions on Automatic Control, vol. 65, no. 11, 2020.

[39] F. Wang, Y. Miao, C. Li, and I. Hwang, "Attitude control of rigid spacecraft with predefined-time stability," Journal of the Franklin Institute, vol. 357, no. 7, pp. 4212-4221, 2020.

[40] A. J. Muñoz-Vázquez, J. D. Sánchez-Torres, S. GutiérrezAlcalá, E. Jiménez-Rodríguez, and A. G. Loukianov, "Predefined-time robust contour tracking of robotic manipulators," Journal of the Franklin Institute, vol. 356, no. 5, pp. 2709-2722, 2019.

[41] A. Lopez, G. Gutierrez, and E. R. Jiménez, "On predefinedtime consensus protocols for dynamic networks," Journal of the Franklin Institute, vol. 357, no. 16, pp. 11880-11899, 2019.

[42] J. K. Ni, L. Liu, and M. Chen, "Fixed-time disturbance observer design for Brunovsky systems," IEEE Transactions on Circuits and Systems II: Express Briefs, vol. 65, no. 3, pp. 341-345, 2017.

[43] F. Yang, C. Z. Wei, and W. Rong, "Fixed-time convergent disturbance observer for first-order uncertain system," Control and Decision, vol. 34, no. 5, pp. 917-926, 2019.

[44] P. Andrey and L. Fridman, "Stability notions and Lyapunov functions for sliding mode control systems," Journal of the Franklin Institute, vol. 351, no. 4, pp. 1831-1865, 2014.

[45] L. Zhang, X. Z. Ju, and N. G. Cui, "Ascent control of heavy-lift launch vehicle with guaranteed predefined performance," Aerospace Science and Technology, vol. 110, pp. 1-18, 2021.

[46] L. Zhang, C. Z. Wei, and L. Jing, "Fixed-time sliding mode attitude tracking control for a submarine-launched missile with multiple disturbances," Nonlinear Dynamics, vol. 93, no. 4, pp. 2543-2563, 2019.

[47] M. Tomas, E. Moulay, and W. Perruquetti, "Fixed-time observer with simple gains for uncertain systems," Automatica, vol. 81, pp. 438-446, 2017. 